\title{
Sicherheit in Zeiten der Pandemie
}

\author{
Bis zum Vorjahr ging es im Sicherheitsmanagement von Österreichs \\ Krankenanstalten vorrangig um abgängige Patienten, Aggressionsereignisse \\ und Katastrophenvorbereitung. Corona hat auch hier \\ Änderungen gebracht. Erika Pichler
}

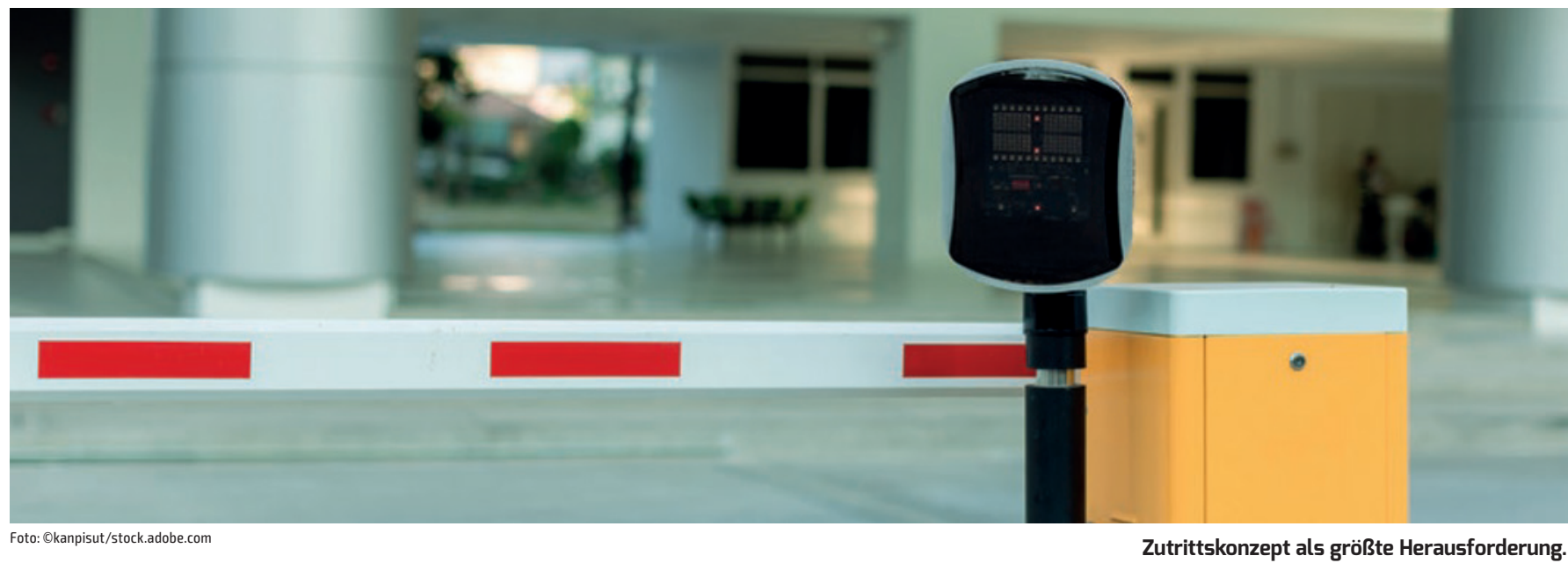

Die Corona-Pandemie hat - wie in so vieler Hinsicht - auch in manchen Bereichen der Spitalssicherheit den Effekt eines Vergrößerungsglases. Waren systematische Zutrittskontrollen an Österreichs Krankenanstalten bisher kaum relevant, wurden sie nunmehr unverzichtbar und werden auch „nach COVID“ möglicherweise Thema bleiben.

„Die größte Herausforderung im Bereich der Sicherheit ist aktuell der geregelte und kontrollierte Zutritt in das Gebäude des Universitätsklinikums AKH Wien, nicht zuletzt aufgrund der Größe des Hauses“, sagt Siegfried Gierlinger, Technischer Direktor am Universitätsklinikum AKH Wien. Generell stehe derzeit aus seiner Sicht im Rahmen der Pandemiebewältigung der Schutz der Mitarbeiter und Patienten im primären Fokus.

Die im Pandemiejahr gesammelten

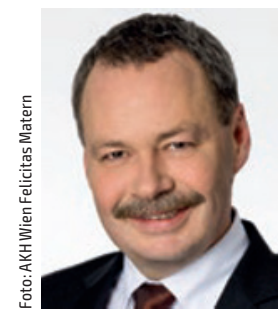
Erfahrungen mit Patienten- und Besucherströmen lassen Gierlinger ein erstes Resümee ziehen: „Der kontrollierte Zutritt und die Besuchsbeschränkungen haben sich auf alle Fälle bewährt. Daher werden derzeit Überlegungen angestellt, für den Normalbetrieb eine Fortführung des geregelten Zutrittes mittels digitaler Unterstützung zu etablieren." Derzeit werde untersucht, „ob eine Zugangskontrolle in ähnlicher Form wie zum Beispiel bei den Flughäfen üblich mit QR-Code, digital oder auf Hardcopy im AKH umsetzbar wäre. Für Mitarbeiter ist hier natürlich der Mitarbeiterausweis entsprechend zu verwenden, und für die Ambulanzpatienten - selbstverständlich mit Ausnahme der Notfälle - muss dafür ein Anmeldesystem flächendeckend umgesetzt werden."

Große Bedeutung bekam am AKH Wien während des letzten Jahres externes Sicherheitspersonal - auch in finanzieller Hinsicht. Die Kosten für eine 24/7-Security an 365 Tagen im Jahr belaufen sich laut Gierlinger schon im Regelbetrieb auf etwa 1,4 Millionen Euro. Aufgrund der durch die Pandemie erforder-

\section{Siegfried Gierlinger, AKH Wien: „Fortführung des geregelten Zutritts im Normalbetrieb."}

lichen Zugangsbeschränkungen entstehen Zusatzkosten von etwa 340.000 Euro pro Monat. Das zusätzlich beschäftigte Sicherheitspersonal unterstützt bei der Zutrittskontrolle und der Triagierung.

\section{Sicherheitsthemen im Normalbetrieb}

Ein für ein Großkrankenhaus typisches Sicherheitsproblem sind abgängige Patienten. Im AKH Wien seien in den letzten Jahren bis inklusive 2019 im Schnitt fünf Patienten pro Woche als ab- 
gängig gemeldet worden, sagt Gierlinger. Die dafür vorgesehenen internen Konzepte des Hauses sowie die notwendige Zusammenarbeit mit der Feuerwache AKH, der Krankenhausbetriebsführung, dem Sicherheitsdienst und der Exekutive hätten sich jedoch sehr bewährt. „Damit wird auch sichergestellt, dass alle Patienten innerhalb kürzester Zeit ausfindig gemacht und wieder einer Behandlung zugeführt werden.“

Die Patientensuche unterliege im AKH Wien nach dem Prinzip der Verantwortung für den Patienten und der damit verbundenen Rechtssicherheit einem dreistufigen Verfahren, sagt Gierlinger. Stufe 1 bedeutet erhöhte Aufmerksamkeit im Rahmen der Routinekontrollgänge, Stufe 2 intensive Suche in den Innen- und Außenbereichen, Stufe 3 den intensivsten Suchprozess mit Ak-

Großereignissen und Katastrophen) und „Sicherheits-Sonderlagen", wie etwa Amokläufe, Bombendrohungen oder terroristische Bedrohungen, zu meistern. Letzteres dürfte ohnehin wegen der Unkalkulierbarkeit derartiger Ereignisse zu den Horrorszenarien jedes Hauses zählen, unabhängig von dessen Größe und Ressourcen.

\section{Maßnahmenbündel gegen Patientenaggression}

Was jedoch aggressives Verhalten als Sicherheitsrisiko betrifft, so setzen die SALK (so wie auch das AKH Wien) auf ein Bündel an Maßnahmen. „Wir versuchen, dieses Problem mit einem Mix aus baulichen, technischen, organisatorischen und personellen Maßnahmen zu lösen“, sagt Hofer. Dazu zählen laut dem Sicherheitsexperten diverse Schulungen für die Mitarbeiter, die ständige Erreichbarkeit eines Mitarbeiters des Sicherheitsdienstes, die laufende Fortbildung der Mitarbeiter des Sicherheitsdienstes und der laufende Ausbau der elektronischen Zutrittskontrolle. Besonders in der Psychiatrie (beispielsweise in den Unterbringungsbereichen) sei ein gut etabliertes System des Zu-Hilfe-Eilens mit einem Personennotrufsystem wesentlich. Weiters sei die Tragepflicht des Dienstausweises eingeführt worden sowie die Meldepflicht bei Sicherheitsvorfällen, um reagieren zu können (die Daten werden seit Jahren in einer Datenbank erfasst); außerdem die Zusatzbewachung bei Patienten, von denen aufgrund ihrer psychischen Erkrankung Gefahr für das Krankenhauspersonal ausgeht, sowie die Videoüberwachung an neuralgischen Stellen. Im

tivierung zusätzlicher Ressourcen wie etwa der Feuerwehr oder von Mitarbeitern der Technischen Betriebsführung. „Oftmals können diese Patienten aber kurzfristig gefunden werden, mit unterschiedlichsten Begründungen - Ambulanz verlassen, um ein Getränk zu holen, oder auch sich selbst entlassen. Ansonsten wird die zweite Stufe aktiviert beziehungsweise in sehr wenigen Fällen auch die dritte Stufe." Im Jahr 2020 habe es etwa von 221 gemeldeten Patienten nur für 13 der Hinzuziehung zusätzlicher (Einsatz-)Kräfte bedurft.

Ebenfalls in den Aufgabenbereich des Sicherheitsmanagements an Krankenhäusern fällt das Thema Patientenaggression. Im AKH Wien finden regelmäßige klinik- und abteilungsbezogene Deeskalationstrainings statt, die von dafür ausgebildeten AKH-eigenen Trainern abgehalten werden. Auch wurde in $\mathrm{Zu}$ sammenarbeit von Mitarbeitern des Universitätsklinikums AKH Wien und der MedUni Wien ein übergreifendes Sicherheitsboard implementiert, in welchem sicherheitsrelevante Themen (wie auch Aggressionsereignisse) behandelt werden und gemeinsame Lösungsansätze erarbeitet werden.

Die Gewichtung von Sicherheitsproblemen fällt je nach dem Profil einer Krankenanstalt unterschiedlich aus. Zunehmende Aggressivität beziehungsweise das Anspruchsdenken der Patienten und auch der Besucher werden zum Beispiel von Klaus Offner, dem Wirtschaftsdirektor der SALK, sowie deren Sicherheitsexperten Michael Hofer an erster Stelle genannt, wenn nach der aktuell größten Herausforderung im Sicherheitsbereich gefragt wird. An zweiter Stelle fühle man sich durch die Notwendigkeit herausgefordert, sogenannte OGK-Situationen (Organisation bei
Wesentlichen seien alle diese Punkte bereits umgesetzt worden, sagt Hofer. Bei den elektronischen Zutrittskontrollen erfolge ein fortwährender Ausbau.

Klar sei auch, dass die stark frequentierten SALK-Kliniken im Zentralraum einen anderen Sicherheitsbedarf hätten als die dislozierten Landeskliniken im Tennengau, Pongau und Lungau. „Ein Sicherheitskonzept kann nicht mit einem Anzug von der Stange verglichen werden. Ein Sicherheitskonzept ist immer ein Maßanzug. Es muss an die jeweiligen Bedrohungen, Gefährdungen und Umstände angepasst werden“, sagt Offner. „Die Situation in der Landesklinik Tamsweg, in dem jeder Mitarbeiter fast alle anderen Mitarbeiter kennt, ist völlig anders als im Uniklinikum."

Was die finanziellen Aufwendungen für den Bereich Sicherheit betrifft, so hatten auch die SALK wegen der COVID-19-Pandemie starke Steigerungen für zusätzlich aufgestocktes Sicherheits- und Einlasspersonal zu verzeichnen. Aus diesem Grund werden sich die Kosten für den Bereich Sicherheit, die sich für Dienstleistungen wie Sicherheitsdienst, Portierunterstützung und Verkehrswächter ansonsten mit rund 600.000 Euro pro Jahr belaufen, um das etwa 2,7-Fache erhöhen. Im Konkreten erfolgt die Sicherheitsüberwachung an den Eingängen durch den Österreichischen Wachdienst (ÖWD). Das Personal für Temperaturkontrolle und Symptomabfrage, das derzeit durch den Arbeiter-Samariter-Bund gestellt wird, wird nach Beendigung der COVID-Krise zur Gänze wieder abgebaut werden.

\section{DR. ERIKA PICHLER}

pichler@gesundheitswirtschaft.at 